\title{
Phonons and Free Carriers in a Strained Hexagonal GaN-AIN Superlattice Measured by Infrared Ellipsometry and Raman Spectroscopy
}

\author{
M. Schubert ${ }^{1,2}$, A. Kasic ${ }^{2}$, T.E. Tiwald ${ }^{1}$, J.A. Woollam ${ }^{1}$, V. Härle ${ }^{3,4}$, F. Scholz ${ }^{3}$ \\ ${ }^{1}$ Center for Microelectronic and Optical Materials Research, University of Nebraska, \\ Lincoln, NE 68588-0511, U.S.A. \\ ${ }^{2}$ Abteilung Halbleiterphysik, Institut für Experimentelle Physik II, Universität Leipzig, \\ Vor dem Hospitaltor 1, D-04103 Leipzig, Germany \\ 3 4. Physikalisches Institut, Universität Stuttgart, Pfaffenwaldring 57, \\ D-70569 Stuttgart, Germany \\ ${ }^{4}$ now with Osram Opto Semiconductors, Wernerwerkstr., D-93049 Regensburg, \\ Germany
}

ABSTRACT

Phonon and free-carrier effects in a strained hexagonal $(\alpha)\{\mathrm{GaN}\}_{l^{-}}\{\mathrm{AlN}\}_{m}$ superlattice (SL) heterostructure $(l=8 \mathrm{~nm}, m=3 \mathrm{~nm})$ are studied by infrared spectroscopic ellipsometry (IRSE) and micro $(\mu)$-Raman scattering. Growth of the heterostructures was performed by metal-organic vapor phase epitaxy (MOVPE) on (0001) sapphire. An unstrained $1 \mu \mathrm{m}$-thick $\alpha$-GaN layer was deposited prior to the SL. SL phonon modes are identified combining results from both IRSE and $\mu$-Raman techniques. The shift of the GaN-sublayer phonon modes is used to estimate an average compressive SL stress of $\sigma_{x x} \sim-4.3 \mathrm{GPa}$. The IRSE data reveal a free-carrier concentration of $n_{e} \sim$ $5 \times 10^{18} \mathrm{~cm}^{-3}$ within the undoped SL GaN-sublayers. According to the vertical carrier confinement, the free-carrier mobility is anisotropic, and the lateral mobility $\left(\mu_{\perp} \sim 400\right.$ $\mathrm{cm}^{2} / \mathrm{Vs}$, polarization $E \perp c$-axis) exceeds the vertical mobility $\left(\mu_{\|} \sim 24 \mathrm{~cm}^{2} / \mathrm{Vs}, E \| c\right)$ by one order of magnitude.

\section{INTRODUCTION}

Strain plays an important role for group-III nitride materials because of large lattice mismatch between binary alloys, and most substrate materials available so far [1]. A possible way to overcome difficulty in growth of high-quality $\mathrm{GaN} / \mathrm{Al}_{x} \mathrm{Ga}_{1-x} \mathrm{~N}$ heterostructures may be the growth of superlattice (SL) structures with few Angstrom periods. Such SL's may be highly strained. If the SL period remains within the critical thickness of the SL constituents, the stacked $\{\mathrm{GaN}\}-\left\{\mathrm{Al}_{x} \mathrm{Ga}_{1-x} \mathrm{~N}\right\}$ sublayers should form tensile and compressive stressed "barriers" and "wells" within the SL, and adopt a common in-plane lattice constant. Besides strain-induced shift of SL phonon modes and band-gap energies, the $\mathrm{Al}_{x} \mathrm{Ga}_{1-x} \mathrm{~N}$ sublayers should act as barriers and confine free carriers to the GaN sublayers. These carriers should then behave similarly to a two-dimensional carrier gas because of the mobility confinement perpendicular to the SL interfaces.

Gleize et al. have recently studied a strained GaN-AlN-SL with average Al concentration of $48 \%$ using $\mu$-Raman investigations [2]. The SL was grown by mole-cular beam epitaxy. The authors observed strain-induced shifts of the GaN-sublayer phonon modes, but no free-carrier effects were detected. An estimate for the in-plane strain, $\epsilon_{x x}$, 
and the average biaxial stress, $\sigma_{x x}$, was given using elastic constants $\left(C_{i j}\right)$, and phonon deformation potential constants $\left(a_{\lambda}, b_{\lambda}\right)$ for $\alpha$-GaN given in Refs. [3] and [4], respectively.

Spectroscopic ellipsometry (SE) is known to be an excellent technique for measurement of thin-film optical properties. Successful application of infrared (IR) SE for measurement of phonon and free-carrier effects in group-III nitride heterostructures was reported previously [5,6]. SE is an indirect technique, and calculated model spectra need to be fit to experimental data. If appropriately chosen, model parameters, which parameterize the dielectric functions of the sample constituents, can provide physically meaningful quantities, such as transverse-optical (TO), longitudinal-optical (LO) frequencies and broadenings, static or high-frequency dielectric constants, and layer thickness. Because free-carrier absorption affects the IR dielectric response, concentration and mobility parameters can be extracted from the IRSE lineshape analysis if the carrier effective mass is known [7]. When combined with electrical Hall measurements, the IRSE data can further provide information about the effective mass parameter, as recently demonstrated for $n$ - and $p$-type $\alpha$-GaN [6].

The focus of this work is to investigate stress and free-carrier effects in a strained $\alpha$ GaN-AlN-SL by measurement of the long-wavelength dielectric response using IRSE for the first time, in combination with $\mu$-Raman investigations.

\section{EXPERIMENTAL}

One SL with 16 periods of alternating wurtzite $\mathrm{GaN}(8 \mathrm{~nm})$ and $\mathrm{AlN}(3 \mathrm{~nm})$ layers was grown by MOVPE on (0001) sapphire. A 1- $\mu$ m-thick GaN layer was deposited on a $\sim 15$ $\mathrm{nm}$ AIN buffer layer prior to the SL (for more details see Ref. [8]). The sample was measured at room temperature by IRSE and polarized $\mu$-Raman scattering. Ellipsometric parameters were acquired at multiple angles of incidence $\left(57^{\circ}, 72^{\circ}\right)$, and for wavenumbers from $333 \mathrm{~cm}^{-1}$ to $1200 \mathrm{~cm}^{-1}$. A rotating-polarizer, rotating-compensator, Fouriertransform-based variable-angle-of-incidence spectroscopic ellipsometer was used. A detailed description of the IRSE approach is given in Refs. [5,7] and references therein. The $\mu$-Raman spectra were recorded with a XY-Dilor spectrometer at five different configurations in backscattering geometry for wavenumbers from $200 \mathrm{~cm}^{-1}$ to $1200 \mathrm{~cm}^{-1}$. The excitation wavelength was $488 \mathrm{~nm}\left(\mathrm{Ar}^{+}\right.$laser). The incident laser light power was 150 $\mathrm{mW}$. The diameter of the laser focus at the sample surface was typically $1 \mu \mathrm{m}$. The sample orientations during Raman and IRSE measurement are shown in Figs. 1b, and 2b, respectively.

\section{DISCUSSION}

Fig. 1a shows the $\mu$-Raman spectra in five different backscattering configurations. The spectra reveal the $A_{1}(\mathrm{TO}), E_{1}(\mathrm{TO})$ (labeled by " 1 ", and "2", respectively), and $E_{2}, A_{1}(\mathrm{LO})$, and $E_{1}(\mathrm{LO})$ phonon modes of the $1-\mu \mathrm{m}$-thick $\mathrm{GaN}$ layer which supports the SL. 


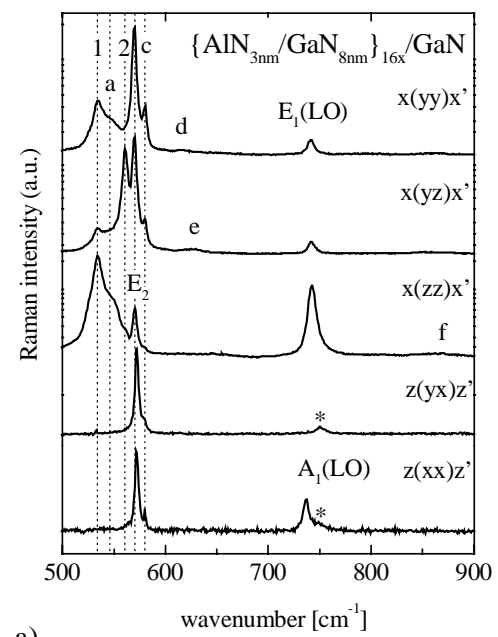

a)

Figure 1. a) Room-temperature $\mu$-Raman spectra recorded in different backscattering configurations from the AlN-GaN-SL sample (sapphire modes are marked by asterisks). b) Sample setup and directions used for the Porto notations.

The phonon frequencies are summarized in Table I. For convenience, we follow the same notation as used by Gleize et al., and label the additional Raman features within Fig. 1a. at $549,580,616,630$, and $860 \mathrm{~cm}^{-1}$ by $a, c, d, e$, and $f$, respectively [2]. By changing the position of the Raman beam focus from the sapphire interface toward the sample surface we observed that all of these features are due to the SL. The most prominent line is $c$ at $580 \mathrm{~cm}^{-1}$, which obeys the symmetry behavior of an $E_{2}$ mode. Its frequency is close to that of the thick GaN layer. A very similar line was observed by Gleize et al. at $587 \mathrm{~cm}^{-1}$, and was identified as the GaN-sublayer $E_{2}$ mode. We also assign $c$ to $E_{2}$ of the SL GaNsublayers. The Raman signal $a$ could belong to a transverse mode with $A_{1}$ symmetry. No clear assignment was done in Ref. [2]. A possible origin for this mode might be the $A_{1}$ (TO) phonon of the AlN-sublattice, but further proof is necessary at this point. Note that we miss the feature labeled $b$ in Ref. [2]. This line was assigned as the SL GaNsublayer $E_{1}(\mathrm{TO})$ frequency. As will be discussed below, according to our SL $E_{2}$ mode observation at $580 \mathrm{~cm}^{-1}$, the SL $E_{1}(\mathrm{TO})$ mode should appear $\sim 570 \mathrm{~cm}^{-1}$, and could therefore be subsumed into the strong $E_{2}$ Raman feature of the thick GaN layer at $570 \mathrm{~cm}^{-}$ ${ }^{1}$. The feature labeled $d\left(616 \mathrm{~cm}^{-1}\right)$ was observed at $620 \mathrm{~cm}^{-1}$ in Ref. [2]. It was suggested that this mode may belong to interface excitations. The Raman signal $e$ at $630 \mathrm{~cm}^{-1}$ in Fig. 1a is identified from the IRSE data analysis as the $E_{1}(\mathrm{TO})$ phonon of the AlN-sublattice (see below). This mode (e) was observed at $635 \mathrm{~cm}^{-1}$ in Ref. [2], but not assigned to a particular sample constituent. The broad and weak structure labeled $f$ is due to a longitudinal mode with $E_{1}$ symmetry. Assignment for this mode from the Raman spectra alone is difficult. However, the IRSE analysis proves that this mode belongs to the highfrequency coupled LO-plasmon-phonon $\left(\mathrm{LPP}^{+}\right)$mode of the SL GaN-sublayers. The corresponding $\mathrm{LPP}^{+}$mode with $\mathrm{A}_{1}$ 


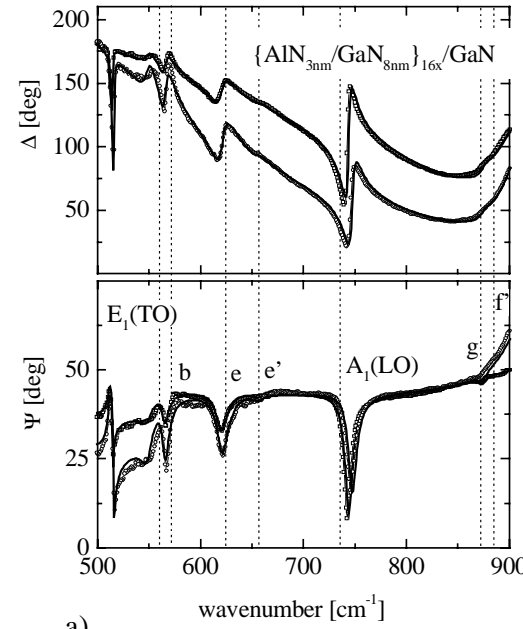

a)

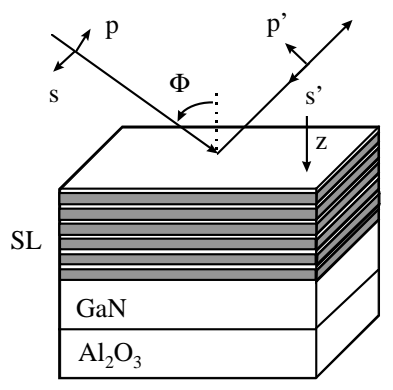

$\rho \equiv \frac{r_{p}}{r_{s}}=\tan \Psi \exp i \Delta$

b)

Figure 2. IRSE data (symbols: experiment; solid lines: best fit) at $57^{\circ}\left(\right.$ ) and $72^{\circ}$ (1) angle-of-incidence $\Phi$ from the AlN-GaN-SL sample. b) Sample setup for the ellipsometric measurement, and definition of $\Psi$ and $\Delta . r_{p}$ and $r_{s}$ are the complex reflection coefficients for parallel $(p)$ and senkrecht $(s)$ polarized light.

symmetry is not observed because of the strong damping of this axial mode due to the vertical confinement of the carriers to the GaN-sublayers (see discussion below).

Fig. 2a presents the IRSE data together with the best-fit model calculation. As discussed somewhere else in detail $([5,6,9])$ for wurtzite III-nitride films on $c$-plane sapphire, the IRSE data provide less sensitivity to transverse modes with $A_{1}$ symmetry. $A_{1}(\mathrm{LO})$ and $E_{1}(\mathrm{TO})$ modes can be identified immediately from resonance structures within the spectra (see Fig. 2a). The $E_{1}(\mathrm{LO})$ modes follow from lineshape analysis of the IRSE data. The best-fit parameters, and those which were not varied during the regression analysis (values in square brackets), are given in Table I. The $E_{1}(\mathrm{TO})$ and $A_{1}(\mathrm{LO})$ frequencies of the "thick" GaN layer are marked within Fig. 2a. The best-fit for the corresponding $E_{1}(\mathrm{LO})$ frequency resulted in $742 \mathrm{~cm}^{-1}$. These results agree excellently with those obtained from the $\mu$-Raman spectra, and compare well with the known $\alpha$-GaN modes [1]. The IRSE data are not sensitive to the IR-inactive $E_{2}$ modes. Hence, the resonance at $570 \mathrm{~cm}^{-1}$ ( $b$ in Fig. 2a) can be immediately identified as the SL GaN-sublayer $E_{1}(\mathrm{TO})$ mode. Furthermore, the feature labeled $e$ in Fig. 1a proves now as a TO resonance ( $e$ in Fig. 2a). It turns out from the IRSE lineshape model calculations that this resonance belongs to the SL AlN-sublayer system. We therefore assign $e$ to the $E_{1}(\mathrm{TO})$ mode of the AlN-sublayers. The small dip at $875 \mathrm{~cm}^{-1}(g)$ is further assigned to the $A_{1}(\mathrm{LO})$ mode of the SL AlN-sublayer system. The weak features at $658 \mathrm{~cm}^{-1}\left(e^{\prime}\right)$ and $880 \mathrm{~cm}^{-1}\left(f^{\prime}\right)$ are identified as the $E_{1}(\mathrm{TO})$ and $A_{1}(\mathrm{LO})$ modes of the AlN buffer layer, respectively. Gleize $e t$ al. [2] used the observed shift of the SL GaN phonon modes to calculate the average biaxial stress $\sigma_{x x}$ and strain $\epsilon_{x x}$ within the SL structure. The elastic coefficients $C_{i j}$ and phonon deformation potential constants $\left(a_{\lambda}, b_{\lambda}\right)$ for $\alpha-\mathrm{GaN}$ 
Table I: Phonon mode frequencies of the GaN-AlN-SL in $\mathrm{cm}^{-1}$. Values in square brackets were not varied during the IRSE analysis.

\begin{tabular}{|c|c|c|c|c|c|}
\hline & $A_{1}(\mathrm{TO})$ & $E_{1}(\mathrm{TO})$ & $E_{2}$ & $A_{1}(\mathrm{LO})$ & $E_{1}(\mathrm{LO})$ \\
\hline $\begin{array}{c}\text { GaN } \\
\text { "buffer" }\end{array}$ & $\begin{array}{l}534^{a} \\
534^{b}\end{array}$ & $\begin{array}{l}560^{a} \\
560^{b}\end{array}$ & $570^{a}$ & $\begin{array}{l}737^{a} \\
737^{b}\end{array}$ & $\begin{array}{l}742^{a} \\
742^{b}\end{array}$ \\
\hline $\begin{array}{c}\mathrm{GaN} \\
\text { Sublayer }\end{array}$ & $\begin{array}{c}-^{c} \\
{[534]^{b}}\end{array}$ & $\begin{array}{c}-^{c} \\
570^{c}\end{array}$ & $580^{a}$ & $\begin{array}{c}-^{c} \\
840^{b}\end{array}$ & $\begin{array}{l}860^{a} \\
840^{b}\end{array}$ \\
\hline $\begin{array}{c}\text { AlN } \\
\text { Sublayer }\end{array}$ & $\begin{array}{l}(549)^{d} \\
{[549]^{d}}\end{array}$ & $\begin{array}{l}630^{a} \\
625^{c} \\
\end{array}$ & $-_{-b}^{b}$ & $\begin{array}{c}-^{b} \\
875^{b} \\
\end{array}$ & $\begin{array}{c}-^{b} \\
{[880]^{b}}\end{array}$ \\
\hline $\mathrm{GaN}^{e}$ & $531.8^{e}$ & $559^{e}$ & $568^{e}$ & & \\
\hline $\begin{array}{l}\text { aman } \\
\text { RSE } \\
\text { ot detectal } \\
\text { ntative mo }\end{array}$ & & & & & \\
\hline
\end{tabular}

were taken from Refs. $[3,4]$. Here we find that the $\mathrm{GaN}$-sublayers are subject to an average compressive stress of $\sigma_{x x} \sim-4.3 \mathrm{GPa}$ if we consider the shift of $\sim 11 \mathrm{~cm}^{-1}$ for the $E_{1}$ (TO) mode. This is consistent with the shift of the $E_{2}$ mode $\left(\sim 12 \mathrm{~cm}^{-1}\right.$, see Table I). The GaN-sublayer $A_{1}(\mathrm{TO})$ mode should then appear at $\sim 537 \mathrm{~cm}^{-1}$. The IRSE data are not sensitive to this mode. Unfortunately, we could not find clear evidence of this mode within our $\mu$-Raman spectra. It might be submerged by the stronger $A_{1}(\mathrm{TO})$ mode of the thick GaN layer. (Note that the total thickness of the SL is only $176 \mathrm{~nm}$.) If elastic deformation of the SL is assumed, the average strain for the GaN layers can be calculated using the $C_{i j}$ 's. We obtain here $\epsilon_{x x} \sim-0.9 \%$, which compares well to the in-plane strain (- $\left.0.65 \%\right)$ when both $\mathrm{GaN}$ and $\mathrm{AlN}$-sublayers adopt a common in-plane lattice constant, and if we consider the thickness and the relaxed lattice constants [1] for both constituents. This result suggests that the SL adopts a different in-plane lattice constant than the thick GaN "buffer" layer. We note that Gleize et al., who investigated a SL with average Al concentration of $48 \%$, observed a higher strain $(-1.3 \%)$. This value was also slightly larger than that if a common in-plane lattice constant $(-1.1 \%)$ is assumed. This might be due to uncertainty of the $C_{i j}$ 's known so far.

As a unique feature of the IRSE technique, the ellipsometry parameters are sensitive to free-carrier affected changes within the dielectric functions of thin films. In this work we observe that the SL GaN-sublayers contain free carriers. In particular, we obtain from the best-fit regression analysis that both $\mathrm{LO} A_{1}$ and $E_{1}$ modes are coupled to plasmon excitations, and that the "zero's" of the GaN-sublayer dielectric functions are shifted to higher frequencies [5,7]. To quantify carrier concentration and mobility values, the effective mass parameter has to be known. We assume that the carriers within the GaNsublayer system are free electrons, which may originate from the AlN sublayer system, i.e., from interface and defect induced donor states. Similar carrier effects were observed in unintentionally-doped $\mathrm{Al}_{x} \mathrm{Ga}_{1-x} \mathrm{~N}$ layers [5]. We further assume an isotropic effective mass, and choose a value of $m * / m_{e}=0.22$. [1] As a result, we obtain a carrier concentration of $n_{e} \sim 510^{18} \mathrm{~cm}^{-3}$, and a strongly anisotropic carrier mobility. The lateral (electric field polarization $E \perp c)$ mobility $\mu_{\perp} \sim 400 \mathrm{~cm}^{2} / \mathrm{Vs}$ exceeds the vertical mobility $\left(\mu_{\|} \sim 24 \mathrm{~cm}^{2} / \mathrm{Vs}\right)$ by more than one order of magnitude. Note that for "thick" films we observe that in 
general $\mu_{\|} \geq \mu_{\perp}$ due to the columnar $\mathrm{Al}_{x} \mathrm{Ga}_{1-x} \mathrm{~N}$ film growth [1,5,6]. In Ref. [5] we obtained an isotropic mobility of $\sim 100 \mathrm{~cm}^{2} / \mathrm{Vs}$ for a $535 \mathrm{~nm}$ thick $\mathrm{Al}_{0.28} \mathrm{Ga}_{0.72} \mathrm{~N}$ film. The SL result suggests that the free carriers are limited in their mobility perpendicular to the SL interfaces. This may find a simple explanation because of the lateral carrier confinement by the AlN-sublayers. The anisotropic mobility further explains why the $\mathrm{LPP}^{+}$ mode with $A_{1}$ symmetry cannot be seen whereas that with $E_{1}$ symmetry can be observed within the $\mu$-Raman spectra. It is well known that strong damping of LPP modes hinders their observation by Raman spectroscopy [10].

To summarize, we investigated a GaN-AIN SL structure by IRSE and polarized $\mu$ Raman scattering. We assigned SL phonon modes by combining results from both techniques. The shift of the GaN sublayer phonon modes allowed us to calculate the average biaxial strain within the SL. The so obtained strain value agrees well with the strain if a common SL in-plane lattice constant is assumed. This study shows strong anisotropic optical carrier effects within the GaN-sublayers. The carriers have very high mobility values for movement parallel to the SL interfaces, and are almost confined for movement parallel to the SL growth direction.

\section{ACKNOWLEDGEMENT}

Research is supported in part by DFG contract Rh 28-3/1, and in part by NSF contract DMI-9901510.

\section{REFERENCES}

1. J. W. Orton, Rep. Prog. Phys. 61, 1 (1998).

2. J. Gleize, F. Demangeot, J. Frandon, M. A. Renucci, F. Widmann and B. Daudin, Appl. Phys. Lett. 74, 703 (1999).

3. A. Polian, M. Grimsditch and I. Grzegory, J. Appl. Phys. 79, 3343 (1996).

4. V. Yu. Davydov, N. S. Averkiev, I. N. Goncharuk, D. K. Nelson, I. P. Nikitina, A. S. Polkovnikov, A. N. Smirnov, M. A. Jacobson, and O. K. Semchinova, J. Appl. Phys. 82, 5097 (1997).

5. M. Schubert, A. Kasic, T. E. Tiwald, J. Off, B. Kuhn, F. Scholz, MRS Internet J. Nitride Semicond. Res. 4, 11 (1999).

6. A. Kasic, M. Schubert, S. Einfeldt, D. Hommel, unpublished.

7. T. E. Tiwald, J.A. Woollam, S. Zollner, J. Christiansen, R. B. Gregory, T. Wetteroth, S. R. Wilson and A. R. Powell, Phys. Rev. B 60, 11464 (1999).

8. F. Scholz, V. Härle, H. Bolay, F. Steuber, B. Kaufmann, G. Reyer, A. Dörnen, O. Gfrörer, S.-J. Im, A. Hangleiter, Solid State Electron. 41, 141 (1997).

9. M. Schubert, T. E. Tiwald and C. M. Herzinger, Phys. Rev. B 61 (March 15. 2000).

10. T. Kozawa, T. Kachi, H. Kano, Y. Taya, M. Hashimoto, N. Koide and K. Manabe, J. Appl. Phys. 75, 1098 (1994). 